\title{
Planes nacionales de desarrollo y management público en Colombia (1958-2018). Un estudio bajo la técnica cualitativa del análisis de contenido
}

\section{National development plans and public management in Colombia (1958-2018). A study under the qualitative technique of content analysis}

DOI: http://dx.doi.org/10.17981/econcuc.39.2.2018.08

Artículo de investigación. Fecha de recepción: 21/08/2018 Fecha de aceptación: 20/11/2018

\author{
Rafael Vergara Varela \\ Universidad del Valle (Colombia) \\ rafael.vergara@correounivalle.edu.co
}

Para citar este artículo:

Vergara, R. (2018). Planes nacionales de desarrollo en Colombia y management público (1958-2018). Un estudio bajo la técnica cualitativa del análisis de contenido. Económicas CUC, 39(2). 129-144. DOI: http://dx.doi.org/10.17981/econcuc.39.2.2018.08

\section{Resumen}

Este artículo presenta una serie de reflexiones académicas e investigativas en torno al significado y relevancia del concepto management público. A partir de esta conceptualización la investigación se centró bajo la metodología cualitativa de la técnica del análisis de contenido aplicada a los planes nacionales de desarrollo en Colombia, con una lectura holística de los sectores agropecuario e industrial en los últimos 60 años. Las contribuciones y evidencias generadas como resultado de esta investigación, están orientadas a explicar de manera hermenéutica la noción más ajustada de la gestión pública, como un elemento de la gestión pública de la planificación del desarrollo económico, que se inscribe en cada plan de desarrollo que también corresponde a cada uno de los planes. Las administraciones presidenciales en Colombia durante el período delimitado.

Palabras clave: Management público, planes nacionales de desarrollo en Colombia, administración pública, investigación cualitativa, análisis de contenido.

\section{Abstract}

This article presents a series of academic and investigative reflections on the meaning and relevance of the public management concept. From this conceptualization, the research was focused on the qualitative methodology of the content analysis technique applied to the national development plans in Colombia, with a holistic reading of the agricultural and industrial sectors in the last 60 years. The contributions and evidences generated as a result of this research are oriented to hermeneutically explain the more adjusted notion of public management, as an element of public management of economic development planning, which is inscribed in each development plan that also corresponds to each of the presidential administrations in Colombia during the delimited period.

Keywords: Public management, national development plans in Colombia, public administration, qualitative research, content analysis. 


\section{Introducción}

En este artículo se socializan los resultados de la investigación doctoral del mismo nombre en el segundo capítulo sobre $m a$ nagement público y planes de desarrollo en Colombia. En un sentido práctico el estudio muestra los avances en resultados y hallazgos sobre el management público y su relación tanto epistemológica como conceptual de la gestión pública de la planificación desde las narrativas inscritas en los planes nacionales de desarrollo en Colombia durante los últimos 61 años. Asimismo, la exposición del documento revela un análisis lingüístico del concepto management, para luego agregar al debate académico y documental el concepto de público.

En esta nueva definición de management público se concreta pedagógicamente una postura lingüística e institucional de carácter difuso y ambiguo. Empero, esto no significa que el término no sea comprensible sino más bien es todo lo contrario, es un elemento técnico, metodológico y de análisis que permite entender desde lo cuantitativo y lo cualitativo como funciona el gobierno y sus entes públicos desde sus prácticas de gestión o administración.

En términos de la presentación, el artículo responde a un contexto algo general hasta lo particular, es decir busca explicar los términos por separado para luego integrarlos y exponer una apreciación conceptual del tema. Vale la pena agregar también que el término management público es un concepto poco estudiado y abordado en el escenario hispano parlante. Además, hasta la fecha hay muy pocas publicaciones que explican este concepto.

Ahora bien, el cuerpo del trabajo aborda cuatro partes conexas y explicativas de manera secuencial que definen el estudio asi: Uno, la definición y el análisis contextual del termino management público. Dos, los albores de la planeación del desarrollo económico en Latinoamérica y en Colombia. Tres, la metodología de la técnica investigativa para abordar desde los documentos de trabajo la hermenéutica del termino management público. Cuatro, los resultados de la investigación. Y por último, las conclusiones de manera lacónica.

Este estudio está escrito para un público en general, no necesariamente experto, pero ávido de conocimiento. Esta investigación responde por demás a una construcción social de conocimiento en clave del dialogo de saberes, donde se piensa no sólo desde lo económico y la gestión pública, sino que dialoga conjuntamente entre lo político, lo administrativo, lo histórico, lo social, lo institucional, etc.

\section{Análisis integral sobre el termino y el contexto de management público}

Básicamente para ilustrar y referenciar el origen de la palabra management se debe precisar que la definición holística del término corresponde a una contextualización de orden histórico. Es decir, en primer lugar, la aparición descriptiva del concepto management emerge del latín manus (mano) cuya definición posteriormente se resignifica en Europa en los siglos XV y XVI en el idioma ingles bajo el término to $m a$ nage que se define como manejar, administrar, gestionar, dirigir, gobernar, etc. De igual forma el idioma francés presentó el concepto ménagement cuya definición implicaba también gestión, administración, dirección o manejo. A partir de estas definiciones primigenias se enriqueció el término to manage a la formación literal de management en el inglés norteamericano en el siglo decimonónico y veinte, el cual lo define como la administración, gestión, manejo, gerencia o dirección de una empresa a través de utilizar los medios para alcanzar los objetivos o fines (Sanabria, 2007; Vergara, 2018). 
Posteriormente, hace un siglo en la aplicación ideológica del primer management o clásico cuyo origen se delinea como científico, se asumió una postura pragmática e instrumental donde el $m a$ nagement como corriente de pensamiento empresarial gravita en función del modelo circular de etapas por medio del planear, decidir, ejecutar y evaluar. Asimismo, esta estructura planificadora de etapas fue homologada por el politólogo Harold Lasswell en 1940 para definir el ciclo de la política pública donde la formulación, la toma de decisiones, la implementación y la evaluación hacen parte del modelo contemporáneo del management público. Como se puede notar, el management como la gestión y/o la administración pública responden a diferentes enfoques analíticos sustentados en diversos epistemes teóricos bajo el contexto de las teorías de la organización (Marchesnay, 1999; Powell \& Dimaggio, 2000; Martinet, 2002; Vergara y Rodríguez, 2016).

Ahora cuando a la palabra management se le adiciona el término público su significación describe variadas acciones en la gestión o en la administración pública. En este escenario, el significado del término público describe literalmente la no rivalidad y la no exclusión en el goce o disfrute de un bien de carácter público, como por ejemplo son los parques, las plazas, las calles o las avenidas. Empero, vale la pena destacar que el significado del término management público responde a un concepto ambiguo o difuso el cual implica diferentes interpretaciones sobre la temática. También el concepto pareciera ser novedoso, pero en esencia está siendo acuñado en los estudios de antropología política, la sociología organizacional, psicología política, los estudios políticos, la administración pública, la economía del sector público y los estudios de las políticas públicas.
Al respecto, el término management publico implica el manejo de lo público, la administración de lo público, o la gestión pública, por ello se resignifica y toma valor en el bureau como organización técnica y empresarial. Asimismo, el management público se concentra en el manual de funciones para la gestión y coordinación de los asuntos del Estado. En otra definición el management público se presenta en la contextualización del policy maker en el diseño y elaboración de las políticas de Estado sobre la base del modelo de la planeación o de la planificación estatal. De igual manera, el management público responde también a la gestión pública de la planificación del desarrollo; en esta orientación se dirigen y se gestionan los activos, ingresos, insumos y recursos del Estado a la sociedad, mediante la relación Estado-Sociedad, "lo cual permite dar respuestas a las necesidades sociales; desde las explicaciones socio-céntricas donde la presión ciudadana determina el andamiaje de la política pública" (Hernández y Chumaceiro, 2018, p. 58).

En los tiempos actuales el management público de postura funcionalista, racionalista y tecnocrática se centra en resolver los problemas de la administración pública mediante algoritmos positivistas e indicadores de gestión y política pública bajo enfoques de eficiencia, eficacia y economía (Nioche, 1994; Guerrero, 1999 y 2004; Echebarría y Mendoza, 1999).

Por su parte, se encuentra también que el management público responde a la orientación de la gestión pública en la planeación del Estado bajo la concepción del desarrollo económico y social, mediante "políticas que se construyen para dar respuestas a necesidades sociales pero desde dinámicas económicas cambiantes y en función del marco capitalista internacional" (Hernández, Chumaceiro y Ravina, 2017, p. 53). Respecto a esto se presenta 
a continuación una síntesis secuencial en la historia mundial institucional moderna en el paradigma de la gestión de la planeación. Asi pues, la noción de planificación o planeación estatal aparece inmersa explícitamente en los trabajos de los economistas clásicos y neoclásicos europeos desde el año de 1770 , donde temas como la producción, el comercio exterior, el demanda, la distribución del ingreso, el sector agropecuario, el sector industrial, la generación de riqueza, la pobreza, el producto interno bruto, etc. hacen parte la proyección del modelo de Estado. En 1880, Otto Von Bismarck planifica un modelo de previsión social en Alemania dando inicio a los modelos de bienestar. Después, en 1917, Rusia a través de una burocracia centralizada como sistema de política económica especial coordina su transición del feudalismo al socialismo entre los años de 1918 a 1931 (Serge, 1947; Hobsbawm y Beltrán, 1991; Chiriboga, 1998; Hobsbawm, 2006; Pflanze, 2014).

Posteriormente, durante la crisis económica global del año de 1929, John Maynard Keynes elabora la política pública estadounidense denominada: "New Deal" en la administración Roosevelt, como plan de desarrollo para apalancar la demanda agregada norteamericana, siendo este el manifiesto de política paradigmática en su obra teórica de "la teoría general de la ocupación, el interés y el dinero". Asimismo, en la Alemania Nacionalsocialista de la década del treinta se diseñó una política de planificación del desarrollo denominada "warfare" (hacer la guerra) como esquema mimético funcionalista de los modelos de intervención estatal para resolver la crisis económica derivada del impacto de la Primera Guerra Mundial y de las clausulas económicas aplicadas a Alemania en el Tratado de Versalles. Por su parte, los países escandinavos después de la Segunda Guerra Mundial desarrollaron los modelos de protección y bienestar social bajo la orientación de gestión pública en la planificación del desarrollo con un énfasis en el Estado benefactor (Keynes, 1943; Meny y Thoenig, 1992; Esping-Andersen y Wolfson, 1996; Kuhnle \& Hort, 2004).

De otra parte, en 1945 después de la Segunda Guerra Mundial se crea una nueva liga de naciones en la ciudad de San Francisco California con el nombre de Organización de las Naciones Unidas (ONU). Este nuevo orden mundial formuló como política la financiación y la reconstrucción europea bajo la directriz del Plan Marshall. Igualmente, el contexto Latinoamericano tendrá su propio Plan Marshall bajo la figura del modelo Cepalino y de la política de la alianza para el progreso. En concreto ambos planes buscaron el desarrollo latinoamericano bajo el acento de mejorar la productividad industrial, desarrollar el campo, buscar una equidad social campo-ciudad y lograr una estabilidad política-institucional en la región (Hogan, 1989; Santa Cruz, 1995; Arias, 2009; Ocampo, 2004; Cueto, Brown y Fee, 2011; Morgenfeld, 2012).

\section{Management público: el inicio de la planeación en Latinoamérica y el influjo internacional para la implementar la técnica de plan de desarrollo en Colombia}

Desdela década delos años cincuenta el continente latinoamericano había evidenciado una serie de problemas socioeconómicos, entre los cuales se encontraban los altos niveles de inflación, poca integración económica y comercial entre los países de la región continental, el desbalance del comercio exterior con saldos negativos en exportaciones, problemas en la generación o creación de bienes de capital explicado por la dependencia de maquinaria e insumos tecnológicos a los mercados mundiales. Igualmente, a nivel social empieza a presentarse una profunda asimetría entre el campo y la ciudad, la cual estaba siendo explicada por el éxodo de campesinos a 
los centros urbanos cercanos dado que el salario en el campo no estaba totalmente institucionalizado. En el caso colombiano esta diáspora poblacional campesina a las ciudades se generó por el fenómeno incontrolable de asesinatos y violencia política en el sector rural. En suma, el desplazamiento campesino al escenario urbano generó otro tipo de necesidades en cuanto a demandas sociales sobre el empleo, salud, ingresos, vivienda, servicios públicos domiciliarios, educación, seguridad, bienestar, etc (Ruy, 1994; Devés, 2000; Torres, 2002).

Bajo este escenario tan complejo la Comisión Economica para América Latina (CEPAL) diseñó un modelo autodenominado Cepalino con cuatro estrategias básicas para lograr un desarrollo en la región: Uno, promover la industrialización sobre la base de sustitución de importaciones. Dos, impulsar la integración regional. Tres, la adopción del keynesianismo como política económica. Cuatro, integrar en las economías latinoamericanas la noción progreso técnico para el desarrollo sector agrícola y el sector industrial. En este modelo el desarrollo agropecuario es un elemento necesario para la Cepal (Fitzgerald, 1998; Thwaites y Castillejo, 2008; Tickner, 2011).

Por otra parte, en Colombia, desde la década de los años veinte, ya se habían realizado misiones internacionales para el buen funcionamiento de la economía y demás instituciones, tal como fue el caso de la misión Kemmerer, en la cual se recomendó la creación un banco central que regulara la política monetaria y financiera en el país (siendo este el origen fundacional del Banco de la República). Asimismo, fue importante la misión del Banco Mundial llamada "Bases de un programa de Fomento para Colombia en 1950" bajo la dirección de Lauchlin Currie. En el plan se recomendaban políticas para el sector agrario, políticas para el desarrollo industrial, políticas para la industria cafetera y en temas de política social. Es más, el aspecto primordial del estudio apuntó a la despolitización de la administración pública para poder implementar las recomendaciones de política económica. Empero, durante la presidencia de Laureano Gómez Castro la propuesta del plan no fue tenida en cuenta (Valencia, 1987; Currie, 1993; Arévalo, 1997; Colmenares, 1998).

Posterior a la dictadura militar del General Rojas Pinilla y de la junta militar se originó en Colombia el acuerdo político hacia la democracia impulsado por el Frente Nacional, donde cada cuatro años el partido liberal y el partido conservador se alternaban el poder hasta un total 16 años en conjunto. En el primer gobierno del Frente Nacional se realizó un estudio por el sacerdote Louis Joseph Lebret sobre las "Condiciones del Desarrollo en Colombia", donde la misión Kemmerer recomendaba la elaboración de planes de desarrollo coordinados por el Estado. Frente a esto, la administración de Alberto Lleras Camargo impulsó la Ley 19 (1958) con el fin de realizar una reforma a la administración pública y de paso crear el Departamento Nacional de Planeación (DNP) y a su vez re-estructurar el Consejo de Política Economica (CONPES). Asi, durante el gobierno de Lleras Camargo específicamente en el año de 1961 se empezó a elaborar el primer plan de desarrollo en Colombia bajo el apoyo decidido de la administración Kennedy y su política exterior a Latinoamérica denominada: "Alianza para el progreso" (Lebret, 1958; Acevedo, 2009; Rojas, 2010).

Ahora bien, como parte de la evidencia y socialización de este artículo de investigación se presenta a continuación la metodología de análisis para luego exponer los resultados en cuanto a la interpretación del concepto management público en los sectores agropecuario e industrial inscritos en cada plan de desarrollo correspondiente a cada uno de los gobiernos y/o administración presidencial en Colombia entre los años 1958 y 2018. 


\section{Métodología}

A través de un análisis hermenéutico del concepto management público, implícito en los planes de desarrollo, mediante la técnica cualitativa de análisis de contenido empleada por Krippendorff (2004), Bardin (2002) y Berelson (1952), se realiza una socialización desde el punto de vista histórico-económico e institucional donde se puede describir e interpretar el imaginario como la impronta de la gestión pública de planificación del desarrollo definida en cada administración presidencial en Colombia desde el origen del primer plan de desarrollo en la administración de presidencial de Alberto Lleras Camargo hasta el actual mandatario Ivan Duque Márquez. En síntesis, esta investigación presenta panorámicamente la evolución del concepto análogo de management público en cada gobierno presidencial en Colombia durante (1958-2018).

En el estudio y la práctica investigativa de los métodos cualitativos, puntualmente la técnica de análisis de contenido, como herramienta que permite develar la intencionalidad de un documento, se procede a centrarse alrededor de un tema, una palabra, un concepto, un párrafo, un docu- mento, un diagrama, una fotografía, una conferencia o cualquier forma de comunicación oral, escrita o visual. Desde estos objetos de análisis se puede interpretar información oculta que una simple lectura no revela. Es decir, bajo el rigor metodológico de esta técnica se puede describir o interpretar el sentido implícito de los documentos estudiados (Berelson, 1952; Krippendorff, 2004).

Para el desarrollo de esta investigación se elaboró una clasificación categorial con el fin de responder a la interpretación de management público desde los documentos de trabajo. Es decir, desde cada plan nacional de desarrollo en Colombia entre 1958 y 2018 se busca develar el término que más responde a management público en el contexto de gestión de la planificación del desarrollo económico en cada administración presidencial (Tabla 1). En este aspecto es importante resaltar que el análisis se realiza teniendo en cuenta dos sectores económicos fundamentales como son las políticas para el sector agropecuario y las políticas para el sector industrial. Por lo tanto, al identificar el modelo económico y las formas con las que opera las reglas del juego desde las narrativas de cada plan se puede interpretar el concepto de gestión de

Tabla 1.

Clasificación, categorías y subcategorias de la investigación

\begin{tabular}{|c|c|c|c|c|}
\hline $\begin{array}{c}\text { Categoría } \\
\text { Principal de } \\
\text { Analisis-Concepto }\end{array}$ & $\begin{array}{c}\text { Marco } \\
\text { Institucional } \\
\text { e Histórico }\end{array}$ & $\begin{array}{l}\text { Sector } \\
\text { Económico }\end{array}$ & $\begin{array}{c}\text { Subcategorias } 1 \\
\text { (instituciones económicas) }\end{array}$ & $\begin{array}{c}\text { Subcategorías } 2 \\
\text { (paradigma económico) }\end{array}$ \\
\hline \multirow{4}{*}{$\begin{array}{l}\text { Gestión } \\
\text { (Management } \\
\text { público) }\end{array}$} & \multirow{4}{*}{$\begin{array}{l}\text { Plan } \\
\text { Nacional } \\
\text { de } \\
\text { Desarrollo } \\
(1960-2018)\end{array}$} & \multirow{4}{*}{$\begin{array}{l}\text { Agropecuario } \\
\text { e Industrial }\end{array}$} & \multirow{2}{*}{$\begin{array}{l}\text { Modelo de Desarrollo } \\
\text { Económico }\end{array}$} & $\begin{array}{l}\text { Economía Cerrada } \\
\text { (Desarrollismo) }\end{array}$ \\
\hline & & & & Apertura Económica \\
\hline & & & \multirow{2}{*}{ Reglas del Juego } & $\begin{array}{l}\text { Intervencionismo } \\
\text { Proteccionismo } \\
\text { (Keynesianismo) }\end{array}$ \\
\hline & & & & $\begin{array}{l}\text { Libre Mercado } \\
\text { Ajuste Estructural } \\
\text { Neoliberalismo } \\
\text { (Economía de Mercado) }\end{array}$ \\
\hline
\end{tabular}

Fuente: Elaboración Propia. 
la planificación del desarrollo económico que más se ajusta a ese management público para cada gobierno presidencial desde 1958 hasta la fecha.

Vale la pena agregar que las fuentes documentales son públicas y se encuentran disponibles en la página del Departamento Nacional de Planeación (DNP). A continuación, se presentan los resultados y los hallazgos de la investigación que permiten responder al significado análogo de management público desde las narrativas documentales teniendo en cuenta el escenario de la gestión de la planificación del desarrollo económico en Colombia.

\section{Resultados}

A manera de síntesis se presentarán los resultados de la investigación que responden a los planes nacionales de desarrollo en Colombia con base en la contextualización del termino (gestión) management público. Respecto a la tabla 1, hay que recordar que el período estudiado corresponde a dos escenarios coyunturales y estructurales (Figura 1).

En relación a la figura 1 se encuentra un escenario de larga duración de 61 años en relación a la implementación de los planes nacionales de desarrollo. En este sentido, Colombia ha trasegado en dos enclaves económicos claramente establecidos. Un primer período que inicia desde 1958 hasta finales de la década los años ochenta donde su modelo económico se concentra en el escenario de una economía cerrada o proteccionista bajo la egida de la sustitución de importaciones cuyo propósito estaba en fortalecer ciertos sectores clave para el desarrollo económico colombiano. En esta orientación de política económica se busca proteger y mejorar la producción nacional en la generación de bienes de consumo y en la producción de bienes intermedios. Empero, la excepción a la norma de la sustitución de importaciones aplicaba solo para bienes de capital y para algunos bienes intermedios o insumos de tecnología necesarios para la producción. Por otra parte, el segundo período económico inicia en Colombia después de 1990 en un esquema de apertura económica sobre la base de desregular el modelo de sustitución de importaciones. Cabe recordar que este modelo del mercado autorregulado es transferido al país por el diseño institucional de las políticas establecidas por los organismos multilaterales como la ONU, FMI, OEA, entre otras.

Igualmente, al interior de esta periodización de 60 años también se logra establecer cuatro subperíodos de análisis que correspondieron a los cambios institucionales que se derivaron del estudio de las narrativas sectoriales inscritas en los planes nacionales de desarrollo y desde los cuales se reinterpreta el concepto implícito $\mathrm{u}$ oculto de gestión de la planificación del desarrollo económico y/o (management público) de cada administración presidencial en Colombia. Estos subperíodos fruto de la investigación y el estudio a los planes obedecen a cuatro puntos de partida cronológicos definidos asi: Uno (1958-1974), autodenominado "frente nacional". Dos (1975-1989), llamado como "transición institucional”. Tres (1990-2001), catalo-

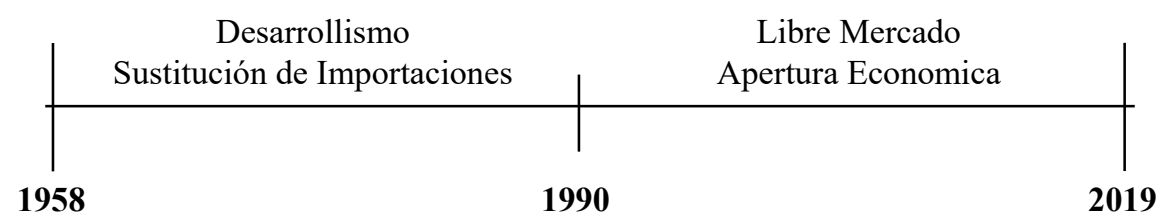

Figura 1. Escenarios económicos en Colombia (1958-2019). 


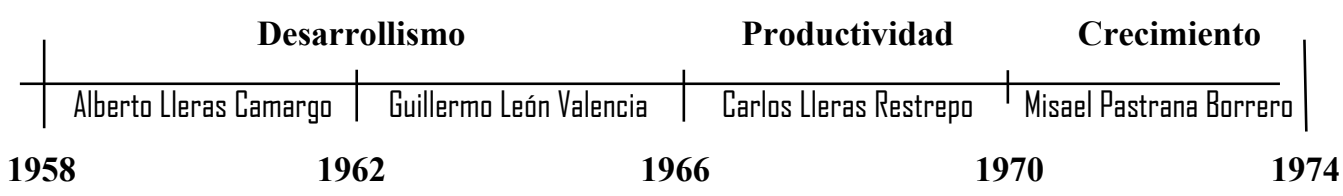

Figura 2. Periodos económicos en Colombia durante el frente nacional (1958-1974)

Fuente: Elaboración propia.

gado como "apertura económica". Y cuatro (2002-2019), establecido como "reprimarización de la economía". En estas cuatro etapas o subperíodos se generaron los puntos de partida que corresponden también al concepto apropiado a management público de cada gobierno presidencial en Colombia.

La primera subetapa (1958-1974) coincide por demás con la coyuntura política en el país del denominado: "frente nacional" (Figura 2). Como bien se explica en la sesión dos de este artículo, entre los años 1953-1958 hubo una dictadura militar en el país. La transición a la democracia se firmó con el acuerdo político del frente nacional. En últimas, esto implicó una alternancia de poder cada cuatro años entre liberales y conservadores. En esta subetapa se generaron los primeros pasos en el diseño, formulación e implementación de los planes nacionales de desarrollo en Colombia.

Vale la pena anotar también que el primer plan de desarrollo se realizó en la administración del presidente Alberto Lleras Camargo donde el plan apuntaba a esta temporalidad (1960-1974) dada la ayuda técnica y financiera de la política: "Alianza para el progreso". En este primer gobierno del retorno a la democracia, la noción de management público se definía como "desarrollismo" porque precisamente su orientación buscaba un equilibrio entre lo económico, lo social y lo institucional. Además, su orientación ideológica se ajustaba en la estructura de las teorías de la modernización. Por esto es relevante el desarrollo en los tres ejes mencionados.
Desde el anclaje económico se esperaba modernizar técnicamente al sector industrial, siendo mirado como una función de producción neoclásica. Asi, tambien se buscó modernizar también al sector agropecuario y sus técnicas de producción a la manera de una empresa exportadora. En últimas, el objetivo de este plan estaba en función de sentar las bases para industrializar el campo colombiano y de paso atenuar el éxodo poblacional del campo a la ciudad sobre la base de generación de empleo en el sector rural. Este plan de desarrollo estuvo vigente entre 1961 y 1968 (DNP, 1961).

Para 1968, en la administración presidencial de Carlos Lleras Restrepo se diseñó un plan de desarrollo económico con un horizonte temporal hasta el año de 1972. La noción de management público en el plan se asimila bajo el concepto de "productividad". Para este plan de desarrollo es central mejorar los niveles de productividad en el sector agropecuario. Por ello, la política agropecuaria se encamina a una reforma agraria donde fortalezca la expansión del crédito y la tecnificación del campo. Y en el sector industrial se buscaba aumentar la productividad en la elaboración de los bienes de consumo, intermedios y de capital. Empero, este plan finaliza en 1971 (DNP, 1969).

En el último cuatrenio del Frente Nacional durante la administración de Misael Pastrana Borrero el concepto management público se define como: "crecimiento". Básicamente esta política funcionaba alrededor de cuatro estrategias de desarrollo urbano, comercio exterior, 


\begin{tabular}{|c|c|c|c|}
\hline Equidad & Integración & Competitividad & Modernización \\
\hline Alfanso López Michelse & Julia Cesar Turbay Ayala & Belisario Betancur Cuartas & Virgilia Barco Vargas \\
\hline
\end{tabular}

Figura 3. Periodo de transición institucional en Colombia (1975-1989).

Fuente: Elaboración propia.

ahorro privado y política social. El argumento central de esta política era generar los medios suficientes para un desarrollo social en el campo como en la ciudad y el eje conductor del crecimiento económico es la construcción como sector líder (DNP, 1971).

En la segunda subetapa (1975-1989) se le denominó como "transición institucional" por las siguientes razones: Primero, este es un período postfrente nacional, es decir pasamos de una democracia restringida a una democracia participativa. Segundo, en este período se observa una transición en el modelo económico del proteccionismo al mercado. Y tercero, hay un cambio institucional donde se impulsa la elección popular de alcaldes y la descentralización político-económica hacia las regiones en el país.

En la administración presidencial de Alfonso López Michelsen (1974-1978), el concepto de management público que emerge de su plan de desarrollo tiende a la noción de "equidad". En un sentido práctico el plan de desarrollo de López buscaba cerrar la brecha es decir disminuir las asimetrías campo-ciudad con el fin de mejorar el bienestar de los territorios. El plan de este gobierno se concentró en políticas focalizadas bajo el enfoque de justicia social donde se buscaba mejorar las condiciones del $50 \%$ más pobre en las urbes y en el sector rural (DNP, 1974).

Para el gobierno Turbay Ayala el concepto que más se asimila a management público es "integración". Es decir, se busca una integralidad tanto para el sector agropecuario como para el sector industrial. La política inscrita en el plan buscaba mejorar la productividad de los sectores económicos. Por ello, el elemento básico de este plan de desarrollo tiende a la descentralización territorial con el ánimo de impulsar otras zonas productivas a las ya establecidas en el país como: Bogotá, Medellín, Cali y Barranquilla (DNP, 1978)

En cambio, en la administración presidencial de Belisario Betancur Cuartas la versión de management público tiende a la "competitividad". En este gobierno se realizan los primeros atisbos para flexibilizar el modelo de sustitución de importaciones. Por esta razón, el plan de desarrollo del presidente Betancur buscaba mejorar la productividad y las condiciones de la producción nacional, con el fin de que el sector industrial pudiera competir en el mercado internacional como una apuesta de mejorar las condiciones de desarrollo (DNP, 1982).

De otra parte, en el gobierno de Virgilio Barco el concepto de gestión o "management público" se aproximaba al término de "modernización económica". La política inmersa en el plan buscaba acercarse a un cambio en el modelo económico, donde las narrativas sectoriales del plan de desarrollo dialogan en función de una nueva apuesta en las lógicas del mercado y de la aldea global tomando como base el comercio internacional. En últimas, lo que se está definiendo en este gobierno es un cambio institucional donde hay establecido un tránsito al libre mercado y una reforma a la administración pública (DNP, 1986). 


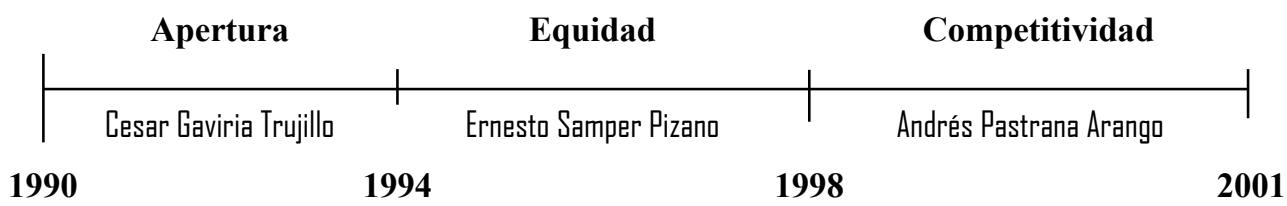

Figura 4. Periodo de ajuste estructural de la economía colombiana (1990-1991).

Fuente: Elaboración propia.

En la tercera subetapa (1990-2001) denominada de apertura económica o ajuste estructural de la economía colombiana se inicia el período de las reformas institucionales. Primero, hay un cambio de constitución política en 1991. Segundo, se le pone fin al modelo de sustitución de importaciones. Y tercero, hay una transformación en la administración pública hacia una agencialización o reorganización. En últimas, hay una reforma política, una reforma institucional y una reforma sectorial.

Durante el gobierno del presidente Cesar Gaviria el concepto de management público que más se ajusta desde su plan de desarrollo es el de "apertura económica". En este período de gobierno se continúa el proceso de modernización de la economía colombiana desgravando aranceles de importación y clausurando el otrora modelo de sustitución de importaciones. En este contexto la acción del estado es marginal es decir empieza a implementarse la acción del Estado mínimo dando por sentado a los sectores productivos que la inversión debe ser más de actores privados. En este escenario empiezan a presentarse los procesos de privatización y terciarización en el sector público (DNP, 1990).

En la administración presidencial de Ernesto Samper Pizano el termino de management público que emerge de las narrativas de su plan de desarrollo se asimila como "equidad". Aunque continúan los procesos de ajuste estructural, este gobierno se centró en el salto social como una manera de proteger a la población más pobre del país; y para ello, crea el Sisben. Empero, en este gobierno los procesos de endoprivatización son más tenues pensando más en el costo social. En relación al desarrollo agropecuario e industrial hubo una mayor protección del Estado que en la administración anterior (DNP, 1994).

Por su parte, el gobierno de Andrés Pastrana y su plan nacional de desarrollo delinea un escenario de management público (gestión de la planificación) que se orientaba a la noción de "competitividad" en función de una economía de exportación. Además, su idea de competitividad va de la mano del proceso de negociación del conflicto en relación al desarme de las guerrillas en un contexto de paz. Sin embargo, en este período se acentuaron los

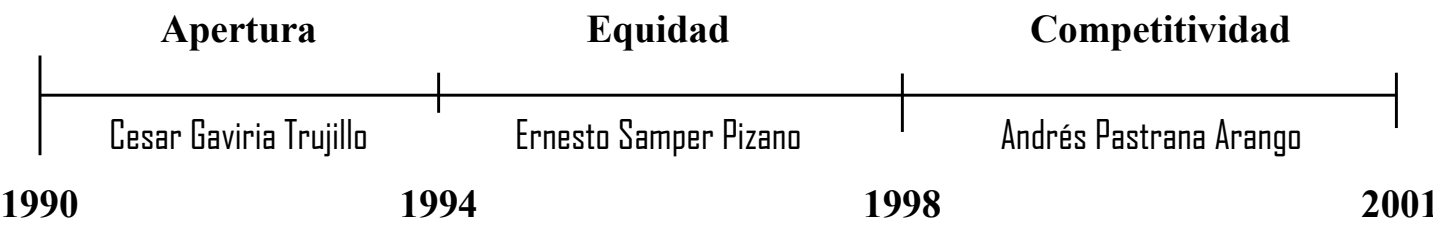

Figura 5. Periodo de reprimarización de la economía colombiana (2002-2022). 
procesos de privatización en el país y la competitividad es asumida como el influjo y la llegada de la inversión extranjera en el país (DNP, 1998).

En este último período (2002-2022) (Figura 5), la tendencia económica es la reprimarización de la economía colombiana donde las apuestas del desarrollo del país se definen en los mercados internacionales en torno a la exploración y explotación de los recursos minero-energético e hidrocarburos. Sin embargo, cada gobierno presidencial tiene sus particularidades en torno al tema sobre el desarrollo económico.

En los dos períodos del gobierno presidencial de Álvaro Uribe Vélez (20022010) sus dos planes de desarrollo describen una versión de management público que se orientaba al "crecimiento económico y a la competitividad". No obstante, la matriz diagonal de estos dos planes se fundamentaban sobre la inminente recuperación de la seguridad democrática como base para mejorar la productividad, el mercado exterior e interno de los sectores económicos del país. Por lo tanto, en la medida en que las fuerzas militares combatían la inseguridad y recuperaban el territorio como la soberanía del Estado de los grupos insurgentes, se mejoraba la calificación de riesgo internacional y con ello el retorno de la inversión extranjera como fenómeno del posicionamiento de la confianza inversionista en el país. Estas acciones afectaban positivamente la competitividad y el crecimiento de la producción nacional (DNP, 2002, 2006).

En cambio, la administración presidencial de Juan Manuel Santos Calderón (2010-2018), en sus dos planes de desarrollo se indica que el término de management público que emerge de las narrativas tiende a la prosperidad económica sobre el énfasis de "ciencia, tecnología e innovación". De manera transversal, a los planes de desarrollo prevalece la construcción de la paz negociada con los grupos guerrilleros como elemento para lograr la estabilidad y la prosperidad. En este aspecto, la paz es un valor agregado para mejorar las condiciones económicas de Colombia. Por ello el crecimiento económico se apoyaba sobre la ciencia, la innovación y la tecnología en el contexto de una locomotora que impulsaba el desarrollo donde la explotación de los recursos minero-energéticos e hidrocarburos son la gran apuesta del país (DNP, 2010, 2014).

Ahora, en el período presidencial de Ivan Duque Márquez la reinterpretación del concepto management público que deviene de la formulación de su plan nacional de desarrollo es "crecimiento económico, productividad y competitividad". Este argumento conceptual de la productividad responde básicamente a fomentar la inversión, la economía naranja, el emprendimiento y las apuestas en el desarrollo de pequeñas y medianas empresas en Colombia. Con estos elementos de conjunto se espera hacerle frente al problema del desempleo. Sin embargo, uno de los aspectos principales del desarrollo del país se expresa en la explotación de los recursos mineroenergéticos e hidrocarburos de la nación (DNP, 2018).

\section{Conclusiones}

El concepto management público responde básicamente a una noción técnica en las prácticas organizacionales de la gerencia pública o de la administración pública como también en el diseño y elaboración de políticas públicas. Aunque pareciera ser un término clásico, su análisis es más bien reciente en lo que implica los estudios de la administración de un gobierno sea 
local, regional o nacional dado este enfoque. Es más, el management público está establecido en el escenario de un policy maker. No obstante, en los términos de este documento, el management público corresponde a la noción de gestión pública de la planificación del desarrollo. Con este estudio no se da por cerrado el debate académico, más bien este documento invita a que se hagan más investigaciones sobre esta temática.

Ahora bien, respecto de los planes nacionales de desarrollo en Colombia y su relación con el management público se pudo evidenciar que esa gestión de la planificación del desarrollo económico está siendo asumida en ideario en el cual históricamente el país está transitando respecto de su paradigma económico o en la coerción institucional que planteen los órganos multilaterales del desarrollo.

En el caso colombiano se pudo establecer cuatro puntos de partida que se conjugaron a nivel internacional. Uno, el surgimiento y apropiamiento de los planes de desarrollo en el contexto del frente nacional bajo el influjo de la política "Alianza para el progreso". Dos, los planes de desarrollo entre (1975-1989) bajo un escenario de transición institucional donde la política tiende de un Estado proteccionista hacia las nuevas tendencias de modernización de la economía sobretodo en la década de los años ochenta a raíz de la crisis internacional de la deuda en la llamada: "década perdida" Latinoamericana bajo influjo institucional del Banco Mundial y del Fondo Monetario Internacional.

Tres, la nueva versión de los planes de desarrollo en Colombia en los años noventa siguen también las nuevas apuestas del desarrollo económico internacional en el cual sus marcos de política apuntaron al libre mercado. Y cuatro, en el nuevo milenio los actuales planes nacionales de desarrollo se han sustentado bajo acuerdos y tratados internacionales donde dialogan en función de la competitividad, la sustentabilidad y la innovación (bajo esquemas de ciencia y tecnología) conjugados especialmente hacia la nueva generación de riqueza de siglo XXI sobre la base de la exploración y explotación de los recursos minieroenergeticos e hidrocarburos.

\section{Referencias}

Acevedo, J. (2009). La planeación nacional y los planes de gobierno: una mirada al desarrollo social, político y económico de Colombia. Revista ciencias estratégicas, 17(22). 291-308.

Arévalo, D. (1997). Misiones económicas internacionales en Colombia 19301960. Historia crítica, (14). 7-24.

Arias, A. (2009). Organismos internacionales y pobreza en América Latina: la matriz de la alianza para el progreso. Revista Electrónica Iberoamericana. 3(1). 11-22.

Bardin, L. (2002). Analisis de Contenido. Madrid: Ediciones Akal.

Berelson, B. (1952). Content analysis in comunication research. Michigan: Free Press.

Chiriboga, M. (1998). Campesinos, utopía y planificación. Revista Ecuador Debate, (15). 231-254.

Colmenares, G. (1998). Historia económica de Colombia. Bogotá, D.C.: Editorial Siglo veintiuno.

Cueto, M., Brown, T. y Fee, E. (2011). El proceso de creación de la Organización Mundial de la Salud y la Guerra Fría. Revista Apuntes, 38(69). 129156. 
Currie, L. (1993). Los objetivos del desarrollo. Cuadernos de Economía. 13(18-19). 163-188.

Devés, E. (2000). El pensamiento latinoamericano en el siglo $X X$ : entre la modernización y la identidad. Buenos Aires: Editorial Biblos.

Echebarría, K. y Mendoza, X. (1999). La especificidad de la gestión pública: el concepto de management público. En, C. Losada, De burócratas a gerentes? Las ciencias de la gestión aplicadas a la administración del Estado. (15-46). Washington, D.C.: Banco Interamericano de Desarrollo.

Esping -Andersen, G. y Wolfson, L. (1996). Después de la edad de oro: el futuro del Estado benefactor en el nuevo orden mundial. Desarrollo económico, 36(142). 523-554. http://dx.doi. org/10.2307/3467359

Fitzgerald, V. (1998). La CEPAL y la teoría de la industrialización. Revista de la CEPAL. (No. Extraordinario: CEPAL cincuenta años). 47-61.

Guerrero, O. (2004). El mito del nuevo Management público. Revista Venezolana de Gerencia, 9(25). 9-52

Guerrero, O. (1999). Una cruzada en pro de la Administración Pública: perspectivas de la ciencia de la Administración Pública desde Iberoamérica. Revista Tecnología Administrativa, 13(29). 29-62.

Hernández, J. y Chumaceiro, A. (2018). Acercamiento histórico a la participación ciudadana en Venezuela: Modelo de relación Estado-Sociedad (19582012). Revista de Ciencias Sociales, 24(2). 56-67.

Hernández, J., Chumaceiro, A. y Ravina, R. (2017). Estado populista y gestión de políticas sociales. Una mirada en américa latina. NEGOTIUM, 38(13). 49-61.
Hobsbawm, E. y Beltrán, J. (1991). Naciones y nacionalismo desde 1780 . Barcelona: Crítica.

Hobsbawm, E. (2006). La era de la revolución, 1789-1848. Barcelona: Editorial Crítica.

Hogan, M. (1989). The Marshall Plan: America, Britain and the Reconstruction of Western Europe, 1947-1952. New York: Cambridge University Press.

Keynes, J. (1943). Teoría general de la ocupación, el interés y el dinero. México, D.F.: Fondo de Cultura EconómicaFCE.

Krippendorff, K. (2004). Content analysis: An introduction to its methodology. (2 ed.). Thousand Oaks, CA: Editorial Sage.

Kuhnle, S. \& Hort, S. (2004). The developmental welfare state in Scandinavia: Lessons for the developing world. Geneva: UNRISD.

Lebret, L. (1958). Estudio sobre las condiciones del desarrollo de Colombia". Bogotá, D. C.: Cromos.

Marchesnay, M. (1999). Diversité des pédagogies de l'entrepreneuriat: l'exemple de Montpellier. J. Fontaine, B. Saporta, T. Verstraete, Actes du 1er Congrès de l'Académie de l'Entrepreneuriat. Entrepreneuriat et enseignement: role des institutions de formation, programmes, méthodes et outtils. (274-285). Lille, Francia. Recuperado de http://thierry-verstraete. com/pdf/actes_1er_congres_AE.pdf

Martinet, A. (2002). Stratégies: actualité et futurs de la recherche. Management International, 6(2), 69.

Meny, I. y Thoenig. J. (1992). Las politicas públicas. Barcelona: Editorial Ariel. 
Morgenfeld, L. (2012). Desarrollismo, Alianza para el Progreso y Revolución Cubana: Frondizi, Kennedy y el Che en Punta del Este (1961-1962). Ciclos en la Historia, la Economía y la Sociedad. 20(40). 133-163.

Nioche, J-P. (1994). Ciencia Administrativa, Management Público y análisis de las políticas públicas: De la evaluación al análisis de las políticas públicas. En, Cuatro lecturas clave sobre políticas públicas. Bogotá, D.C: Pontificia Universidad Javeriana.

Ocampo, J. (2004). El desarrollo económico en los albores del siglo XXI. CEPAL. Bogotá, D.C.: Editorial Alfaomega.

Pflanze, O. (2014). Bismarck and the Development of Germany, Volume II: The Period of Consolidation, 18711880. Princeton, NJ: Princeton University Press.

Powell, W. \& Dimaggio, P. (2012). The new institutionalism in organizational analysis. Chicago: University of Chicago Press.

República de Colombia. Congreso de la República. (25 de noviembre de1958). Sobre reforma administrativa. [Ley 19 de 1958]. DO: 29835.

República de Colombia. Departamento Nacional de Planeación-DNP. (2018). Pacto por Colombia, pacto por la equidad (2010-2014). [Plan nacional de desarrollo. Administración presidencial del Doctor Iván Duque Márquez]. Bogotá, D.C.: DNP.

República de Colombia. Departamento Nacional de Planeación-DNP. (2014). Todos por un nuevo país (2010-2014). [Plan nacional de desarrollo. Administración presidencial del Doctor Juan Manuel Santos Calderón]. Bogotá, D.C.: DNP.
República de Colombia. Departamento Nacional de Planeación-DNP. (2010). Prosperidad para Todos (2010-2014). [Plan nacional de desarrollo. Administración presidencial del Doctor Juan Manuel Santos Calderón]. Bogotá, D.C.: DNP.

República de Colombia. Departamento Nacional de Planeación-DNP. (2006). Estado Comunitario desarrollo para todos (2006-2010). [Plan nacional de desarrollo. Administración presidencial del Doctor Álvaro Uribe Vélez]. Bogotá, D.C.: DNP.

República de Colombia. Departamento Nacional de Planeación-DNP. (2002). Hacia un Estado Comunitario (20022006). [Plan nacional de desarrollo. Administración presidencial del Doctor Álvaro Uribe Vélez]. Bogotá, D.C.: DNP.

República de Colombia. Departamento Nacional de Planeación-DNP. (1998). Cambio para construir la paz (19982002). [Plan nacional de desarrollo. Administración presidencial del Doctor Andrés Pastrana Arango]. Bogotá, D.C.: DNP.

República de Colombia. Departamento Nacional de Planeación-DNP. (1994). El salto social (1994-1998). [Plan nacional de desarrollo. Administración presidencial del Doctor Ernesto Samper Pizano]. Bogotá, D.C.: DNP.

República de Colombia. Departamento Nacional de Planeación-DNP. (1990). La revolución pacífica (1990-1994). Plan nacional de desarrollo. Administración presidencial del Doctor César Gaviria Trujillo. Bogotá, D.C.: DNP.

República de Colombia. Departamento Nacional de Planeación-DNP. (1986). Plan de economía social (1986-1990). [Plan nacional de desarrollo. Administración presidencial del Doctor Virgilio Barco Vargas]. Bogotá, D.C.: DNP.. 
República de Colombia. Departamento Nacional de Planeación-DNP. (1982). Cambio con equidad (1982-1986). [Plan nacional de desarrollo. Administración presidencial del Doctor Belisario Betancur Cuartas]. Bogotá, D.C.: DNP.

República de Colombia. Departamento Nacional de Planeación-DNP. (1978). Plan de integración social (1978-1982). [Plan nacional de desarrollo. Administración presidencial del Doctor Julio César Turbay Ayala]. Bogotá, D.C.: DNP.

República de Colombia. Departamento Nacional de Planeación-DNP. (1974). Para cerrar la brecha (1974-1978). [Plan nacional de desarrollo. Administración presidencial del Doctor Alfonso López Michelsen]. Bogotá, D.C.: DNP.

República de Colombia. Departamento Nacional de Planeación-DNP. (1971). Las cuatro estrategias (1971-1974). [Plan nacional de desarrollo. Administración presidencial del Doctor Misael Pastrana Borrero]. Bogotá, D.C.: DNP.

República de Colombia. Departamento Nacional de Planeación-DNP. (1969). Planes y programas de desarrollo (1969-1972). [Plan nacional de desarrollo. Administración presidencial del Doctor Carlos Lleras Restrepo]. Bogotá, D.C.: DNP.

República de Colombia. Departamento Nacional de Planeación-DNP. (1961). Desarrollo económico y social (1961-1970). [Plan nacional de desarrollo. Administración presidencial del Doctor Alberto Lleras Camargo]. Bogotá, D.C.: DNP.

Rojas, D. (2010). Alliance for progress in Colombia. Revista Análisis político, 23(70), 91-124.

Ruy, M. (1994). La crisis del desarrollismo. En, M. Ruy y M. Millán (Comp.), $L a$ teoría social latinoamericana. Subdesarrollo y dependencia. Tomo II. (135154). México, D.F.: El Caballito.
Sanabria, M. (2007). De los conceptos de administración, gobierno, gerencia, gestión y management: algunos elementos de corte epistemológico y aportes para una mayor comprensión. Revista Universidad \& Empresa, 9(13). 155-194.

Santa Cruz, H. (1995). La creación de las Naciones Unidas y de la CEPAL. $R e$ vista de la CEPAL (57). 17-32.

Serge, V. (1947). Treinta años después de la Revolución Rusa. Páginas ineditas. Recuperado de https://www.revistadelauniversidad.mx/storage/67431767babe-4b12-8969-d1270c630596.pdf

Thwaites, M. y Castillejo, J. (2008). Desarrollo, dependencia y Estado en el debate latinoamericano. Araucaria. Revista Iberoamericana de Filosofía, Política y Humanidades, 10(19). 2445.

Tickner, A. (2011). Relaciones de conocimiento centro-periferia: hegemonía, contribuciones locales e hibridización. En, H. Godoy, R. González y G. Orozco (Eds.), Construyendo lo global: Aportes al debate de relaciones internacionales. (18-34). Barranquilla: Universidad del Norte.

Torres, C. (2002). Grandezas y miserias de la educación latinoamericana del siglo veinte. Buenos Aires: Clacso.

Valencia, J. (1987). La Misión Kemmerer y los intereses financieros británicos en Colombia durante la década de 1920. Lecturas de Economía, (24), 9-48.

Vergara, R. y Rodríguez, M. (2016). El derecho a la educación, al tablero: La política pública de los desayunos escolares en la ciudad de Cali. Revista de Derecho Público, (36). 1-20 http:// d x .doi.org/10.15425/red e pub.36.2016.10 
Vergara, R. (2018). Conceptualización; Administración, Burocracia y Organización, ¿Es el management un sistema adaptativo complejo? Análisis desde las escuelas de pensamiento. Una aproximación preliminar a la temática. Journal encuentros, 17(1). 132-144. http:// dx.doi.org/10.15665/encuent. v17i01.1674
Rafael Vergara Varela es Economista y MSc en Políticas Públicas. Candidato a $\mathrm{PhD}$ en Administración de la Universidad del Valle. Profesor de Políticas Públicas y de Gestión Pública del Programa Académico de Estudios Políticos y Resolución de Conflictos, adscrito al Instituto de Educación y Pedagogía IEP (Univalle). Investigador del grupo: Derecho, Sociedad y Estado, adscrito a la Facultad de Ciencias de la Administración, Universidad del Valle 